elliptic integrals, theta functions, and elliptic functions are taken up in the next three chapters. The relations between the various functions are clearly given and transformations and degenerations are carefully listed.

The two last chapters depart from the treatment of special functions to more general considerations. Chapter VIII treats of integral transforms and their inverses. Included are concise accounts and tables of Fourier, Laplace, Hankel, Mellin, and Gauss transforms. All of these transform relations may be regarded as integral equations of the first kind, with essentially singular kernels and infinite domains of integration. Further analogous integral equations are given, among which are several with finite integration interval, for example, Hilbert's cotangent-kernel equation and Abel's integral equation. Chapter IX is a summary of, for the most part, conventional material on coordinate transformations. A novel feature here is the material on the system of many-dimensional polar coordinates.

Several appendices, one on linear second order differential equations, one on Fourier series, partial-fraction and product representations of some elementary functions, and one on certain summations, complete this comprehensive, scholarly, and useful compilation.

\title{
M. Avramy Melvin
}

Sequential analysis. By Abraham Wald. New York, Wiley, 1947. $12+212$ pp. $\$ 4.00$.

This book could be reviewed from the point of view of the general mathematician, the probability theorist, the mathematical statistician, the theoretical statistician, or from the point of view of any of many kinds of users. We shall try to provide a somewhat composite view.

The central feature of this book is the sequential probability ratio test, abstractly a random walk between adsorbing barriers. We have only to think of a "particle" moving in "steps," the amount of each step being determined by chance in the same way, the stepping process ending whenever the particle, which started from the origin, passes certain "barriers" and leaves an assigned interval. Some 40 pages of the mathematical appendix is devoted to exact and approximate results for such walks. The theory of sequential analysis is growing, and more complex processes are entering; yet this simple random walk is still the core.

To read the body of the book, the reader needs, explicitly, a knowledge of calculus, and, implicitly, a feeling of comfort with sentences 
where the mathematical characters outweigh the punctuation by three to one.

Destructive inspection of military equipment, where every item tested was a scarce item lost to use, called the SPR test into being. Milton Friedman and Allen Wallis's keen appreciation of the need for, and probable value of, sequential methods "gave the incentive for the author's [Wald's] investigations which then led to the development of the sequential probability ratio test" (page 2). This test solved the problem of deciding whether a lot had distribution $A$ or distribution $B$ with given risks of error (there are two ways to be wrong) with the smallest average number of items tested. (The proof that the number of items tested is a minimum was given by Wald and Wolfowitz [1], a year after the appearance of Sequential analysis.)

The solution to this one problem, in the hands of Wald and the other workers at Statistical Research Group, Columbia University, gave usable solutions for testing one mean, or comparing two means, from binomial, normal, and chi-square distributions, where the alternative distributions are really infinite in number, and the true problem is still unmanageable. The theoretical background of these working tests is discussed in considerable and careful detail, as is their connection to the general theory. The practical problem of distinguishing between distributions has learned much from the SPR test, but reducing the average number of trials is not always the consideration; it may be worth more to schedule testing neatly than to reduce it to a minimum. The SRG-C manual on sampling inspection [2], and Wald's later work [3, 4], show some directions in which the subject of sequential testing has broadened out.

In Chapters 4,10 , and 11 , Wald opens a strong attack on an adequate treatment of problems with more than two alternatives. Whenever the alternatives, as in testing composite hypotheses, or the decisions themselves, as in multiple decision problems, are more than two in number, there is a great increase in the difficulty of the problems at the philosophical, theoretical, mathematical, and computational level. This is an important open frontier. Wald's work in these chapters, and his frankly heuristic discussions at appropriate points is of great interest to both the theoretical and the mathematical statistician and should be a continuing stimulus to new work.

In its present form, the book is unsatisfactory for a reader wishing to study the advanced theory of sequential analysis, whether from the point of view of probability or mathematical statistics. Three facts contribute to this: (1) The relegation of the more complex de- 
tails to the appendices, which makes the body of the book accessible to a wider class of readers, has inevitably made a smooth and coherent presentation almost out of the question. (2) The discussion of the various applications are quite repetitive, and while this may be desirable for the general reader, it does not help the mathematician. (3) The book is now two years old, and was written when many parts of the field were undergoing very active development. It is to be hoped that future editions, or a book at a higher level, will provide a more coherent account, covering such new work as that of Wolfowitz [5] and Seth [6] on sequential estimation, Wald [3, 4] and Arrow, Blackwell, and Girshick [7], on multiple decision functions.

The book is relatively free from typographical errors. We may mention that a " $\Delta$ " should appear in the denominator of the fraction appearing at the very end of page 9, that in expression (3.3) on page 38 the inequality should be reversed, that in expression (4.22) on page 85 the fraction on the right should be inverted, and that the summand of the sum in the third line of page 133 should be squared.

\section{REFERENCES}

1. A. Wald and J. Wolfowitz, Optimum character of the sequential probability ratio test, Ann. Math. Statist. vol. 19 (1948) pp. 326-439.

2. Statistical Research Group, Columbia University, Sampling inspection, New York, McGraw-Hill, 1948.

3. A. Wald, Foundations of a general theory of sequential decision functions, Econometrica vol. 15 (1947) pp. 279-313.

4. - Statistical decision functions, Ann. Math. Statist. vol. 20 (1949) pp. 165-205.

5. J. Wolfowitz, The efficiency of sequential estimates and Wald's equation for sequential processes, Ann. Math. Statist. vol. 18 (1947) pp. 215-230. $1-27$.

6. G. R. Seth, On the variance of estimates, Ann. Math. Statist. vol. 20 (1949) pp.

7. K. J. Arrow, D. Blackwell, and M. A. Girshick, Bayes and minimax solutions of seguential decision problems, Econometrica vol. 17 (1949) pp. 213-244.

\section{Melvin P. Peisakoff and John W. Tukey}

Variêtés abéliennes et courbes algébriques. By A. Weil. Paris, Hermann, 1948. $165 \mathrm{pp}$.

This is the second of a series of papers with which the author promised to follow his book, Foundations of algebraic geometry, American Mathematical Society, 1946. The first paper, entitled: Sur les courbes algébriques et les variêtés qui s'en déduisent, is concerned in particular with the theory of correspondences of an algebraic curve 